\title{
A Vortex Method for Wall Bounded Turbulent Flows
}

\author{
Peter S. Bernard \\ Department of Mechanical Engineering \\ University of Maryland \\ College Park, MD 20742, USA
}

\begin{abstract}
Turbulent flow in a channel is simulated using a vortex method. Smoothed vortex sheets cover the near wall region while the vorticity in the remainder of the flow is contained in convecting and stretching vortex filaments. The sheet elements are convected and regridded at each time step thus enabling evaluation of their wall-normal viscous diffusion and vortex stretching using finite differences. New filaments are created during significant ejection events initiated by parent vortices. Chorin's hairpin removal algorithm is used as a physically based subgrid model to limit the range of resolved scales. Calculations on the order of 2000 time steps have been completed thus far. From a perturbed state, the numerical solution develops toward a self-sustained turbulent flow. An initial explosive growth in the number of new vortex structures occurs as the flow compensates for the absence of sufficient spanwise vorticity in the initial conditions. Vortex production is self-limiting, however, and the flow subsequently relaxes toward a quasi-equilibrium in which new vortex filament creation occurs at only a small number of spatially intermittent locations. Preliminary statistics taken from the ongoing simulations suggest that the computational field is approaching the correct physical state.
\end{abstract}

\section{Introduction}

Dynamically interacting, self-replicating quasi-streamwise vortices are a dominant component of the physics of turbulent boundary layer flow [2]. They are responsible, in all likelihood, for the existence of a net turbulent momentum transport near solid walls, i.e. Reynolds stress, which gives turbulent flow its distinctive character. Outward migration of structures created near the wall most likely provides an important source of vorticity populating the outer flow. The effect of viscosity on turbulence at high Reynolds numbers is primarily through wall-normal diffusion of streamwise and spanwise vorticity within a short distance from the wall (typically $y^{+}<30$, where $y^{+} \equiv u_{\tau} y / \nu$ and $u_{\tau}$ is the friction velocity), as well as through dissipation of highly stretched vortices as they convect through the flow.

This picture of the turbulent dynamics of bounded flows strongly suggests the applicability of a vortex method [11] in their simulation. Vortex sheet elements may be used as an economical representation of the high gradient vorticity field near boundaries where primarily one-dimensional viscous vorticity diffusion takes place. At the same time, vortex filaments efficiently model the essentially inviscid interactions of vortex tubes - both coherent or otherwise - populating the region beyond $y^{+}=30$, i.e., beyond the direct influence of viscous wall effects. In a region between the inner and outer flows, provision may be made for the physical processes by which pre-existing coherent quasi-streamwise vortices cause the creation of new structures.

The present work describes the current status in the development of a numerical scheme incorporating these features of turbulent fluid flow. It attempts to capture the essential physics of 
turbulence with minimal resolution and numerical cost. Consistent with this viewpoint, a grid of smoothed vortex sheets is used to represent the near-wall viscous flow while vorticity in the remainder of the domain is contained in vortex filaments. In conformity with previous analyses of the boundary layer structure, new filaments appear in the outer flow during ejection events caused by parent vortices. A preliminary computation of channel flow shows many positive signs of converging toward the physical solution. In particular, both the velocity mean and turbulent structures are consistent with physical experiments and direct numerical simulations.

\section{Vortex sheet and filament scheme}

The evolution of the vorticity field, $\boldsymbol{\Omega}(\mathbf{x}, t)$, in three-dimensional flow is governed by the transport equation

$$
\frac{\partial \boldsymbol{\Omega}}{\partial t}+(\nabla \boldsymbol{\Omega}) \mathbf{u}=(\nabla \mathbf{u}) \boldsymbol{\Omega}+\frac{1}{R} \nabla^{2} \boldsymbol{\Omega}
$$

where $R$ is the Reynolds number, $\mathbf{u}=(u, v, w)$ is the velocity field and $\nabla \mathbf{u}$ is the velocity gradient tensor with components $(\nabla \mathbf{u})_{i j}=\partial u_{i} / \partial x_{j}$. In the present scheme, which is an outgrowth of earlier work $[1,3]$, approximate solutions to (1) are obtained in the form of a grid of vortex sheets or "tiles" confined to the near wall region, together with vortex filaments accounting for significant vorticity away from the boundary as shown in Fig. 1. This arrangement is consistent with the expected extent of non-negligible wall-normal mean vorticity diffusion. In particular, a plot of $d^{2} \bar{\Omega} / d y^{2}$ taken from a spectral channel flow calculation (see Fig. 2) where $\bar{\Omega}$ is the average spanwise vorticity in channel flow, shows that mean viscous diffusion is not significant beyond $y^{+} \approx 35$.

The vortex sheets, of dimension $2 l \times 2 h \times 2 d$ in the streamwise, wall-normal and spanwise flow directions, respectively, have large aspect ratio so that $h<<l, d$. The center of the sheets normal to the wall are located at $y_{i}=(i-1) h, i=1,2, \ldots, N$ so that $y^{*}=(N-.5) h$ is the outer edge of the sheet domain. The vorticity of each sheet is assumed to be uniformly spread over its volume. The layer of sheets with $i=1$ are on the wall and have half thickness. The vorticity of the wall elements is assigned at each time step in such a way as to satisfy the no-slip boundary condition.

In the case of the sheets, (1) is approximated using an explicit scheme in which the vortex stretching and diffusion terms are represented through second order finite differences. The advection term on the left-hand side of (1) is satisfied by convecting the sheets according to the explicit first order formula

$$
\mathbf{x}_{i}^{n+1}=\mathbf{x}_{i}^{n}+\Delta t \mathbf{u}_{i}^{n}
$$

followed by interpolation back into the original sheet locations. Here, (2) approximates the kinematic equation

$$
\frac{d \mathbf{x}_{i}}{d t}=\mathbf{u}\left(\mathbf{x}_{i}(t), t\right)
$$

where $\mathbf{x}_{i}(t)$ is the center of the $i$ th sheet, $\mathbf{x}_{i}^{n}$ is a discrete approximation to $\mathbf{x}_{i}(n \Delta t), \mathbf{u}_{i}^{n} \approx \mathbf{u}\left(\mathbf{x}_{i}^{n}, n \Delta t\right)$ and $\Delta t$ is the time step of the calculation. The linear formula

$$
\boldsymbol{\Omega}(\mathbf{x})=\sum_{j} \gamma_{j} \boldsymbol{\Omega}_{j}
$$

is used for interpolation where $\gamma_{j} \equiv m\left((x-l, x+l) \cap\left(x_{j}-l, x_{j}+l\right)\right) /(2 l) \cdot m\left((y-h, y+h) \cap\left(y_{j}-\right.\right.$ $\left.\left.h, y_{j}+h\right)\right) /(2 h) \cdot m\left((z-d, z+d) \cap\left(z_{j}-d, z_{j}+d\right) /(2 d)\right.$ where $m(S)$ is the rectilinear measure of the set $S$. Thus $\gamma_{j}=1$ when $x=x_{j}, y=y_{j}$ and $z=z_{j}$, while $\gamma_{j}=0$ if $(x-l, x+l) \cap\left(x_{j}-l, x_{j}+l\right)=\emptyset$ and so forth. The sum in (4) is just over those vortices in the immediate neighborhood of a point, so it is of minimal computational cost. 
Whenever $v>0$ near the surface, after application of (2), the sheets with center at $y=h$ will move off away from the boundary creating a gap in the vortex element population. To prevent this from degrading the accuracy of (4), the outer edge of the wall sheets (at $y=h / 2$ ) are moved through application of (2), thus reducing the gap between the sheets near the wall. Appropriate modification of (4) to include variable $h$ is also necessary in this case.

The convective part of the sheet calculation is equivalent to that developed previously [1], with the exception that regridding is now performed at every time step. This maintains a well ordered state of the vortex sheets so that second order finite differences can be used for the stretching and diffusion terms. This approach removes the need to use a deterministic diffusion scheme such as was pursued previously. It also has the advantage of requiring a minimum number of velocity evaluations in accounting for stretching effects.

The filament part of the calculation is adapted directly from the approach of Chorin [5]. Each filament is assigned a constant circulation along its length. The end points of each segment are moved in time according to a discretization of (2). This accounts for both the stretching term in (1) and the convection term. While vortex filament algorithms often use a fourth-order Runge-Kutta scheme to advance the tubes, this is not pursued here in order to reduce the number of velocity evaluations. Since small scale motions are suppressed in the scheme anyway, e.g. through hairpin removal, it is not expected that first order positioning of vortex tubes via (2) should adversely effect the evolving chaotic field.

Folded vortex segments forming hairpins are removed when their interior angle is less than a critical angle [5]. Here we take this to be $45^{\circ}$. Long segments are divided in two whenever they stretch too far, while short segments are joined to larger ones.

Coherent vortex filaments and tubes in a turbulent flow presumably have a finite life span. The demise of such structures as coherent entities may be a natural outcome of their interactions with other vortices, combined with the long time effects of diffusion. The end result is a release of their vorticity into the background, non-coherent vorticity. In the case of a periodic flow, such as a channel, it is particularly important to accommodate the life cycle of coherent vortex tubes since otherwise the numerical scheme will see a monotonic growth in the number of structures as new ones are created. In external flows the problem is much less severe since the destruction of vortices presumably takes place downstream after they have exited the flow domain.

Core spreading was previously introduced $[8,9]$ as a means of modeling viscous diffusion of vortex tubes. While the numerical inconsistencies [6] of this approach may be correctable [12], it is not well suited to the present purposes since it yields a field of ever thickening structures. A more useful approach is to allow vortices which are not being sufficiently stretched to lose an appropriate amount of their circulation at each time step, specifically, that amount which would be contained in the outward diffusing vorticity. Vortices whose circulations drop below a threshold, can then be eliminated.

A model for this phenomenon can be fashioned from the Gaussian core solution [8]. In this, a tube whose length increases from $\Delta s$ to $\Delta s^{\prime}$ in time $\Delta t$ must have a radius equal to

$$
r_{e}=2 \sqrt{\frac{\Delta t}{R_{e}\left(\Delta s^{\prime} / \Delta s-1\right)}}
$$

if the stretching rate is to be in equilibrium with viscous spreading. Generally, it can be expected that tube radii will be inconsistent with the rate of stretching. A tube segment for which $\Delta s^{\prime}>\Delta s$ will thicken if its radius $r<r_{e}$, and a calculation yields that the necessary adjustment of circulation is:

$$
\Gamma^{n+1}=\Gamma^{n}\left(1-\frac{4 \Delta t}{R_{e}}\left(\frac{1}{r^{2}}-\frac{1}{r_{e}^{2}}\right)\right)
$$


If $r \geq r_{e}$, the circulation may be left untouched, i.e.

$$
\Gamma^{n+1}=\Gamma^{n}
$$

If contraction takes place, i.e. $\Delta s^{\prime}<\Delta s$, then the maximum circulation can be assumed to be lost so that

$$
\Gamma^{n+1}=\Gamma^{n}\left(1-\frac{4 \Delta t}{R_{e}} \frac{1}{r^{2}}\right) .
$$

For tubes composed of multiple segments, (6) - (8) may be applied using the average rate of stretching along the tubes.

\section{Velocity field}

Following common practice [11], the velocity field is computed through evaluating the smoothed Biot-Savart integral

$$
\mathbf{u}(\mathbf{x}, t)=\int_{\Re^{3}} K_{\eta}\left(\mathbf{x}-\mathbf{x}^{\prime}\right) \mathbf{\Omega}\left(\mathbf{x}^{\prime}, t\right) d \mathbf{x}^{\prime}
$$

where the local smoothing function, $K_{\eta}$, is used to desingularize the velocity field. In the case of sheets,

$$
K_{\eta}= \begin{cases}K & |\mathbf{x}| \geq \eta \\ K\left(\frac{5}{2}-\frac{3}{2}\left(\frac{\mathbf{x}}{\eta}\right)^{2}\right) \frac{|\mathbf{x}|^{3}}{\eta^{3}} & |\mathbf{x}|<\eta\end{cases}
$$

where

$$
K(x, y, z)=-\frac{1}{4 \pi|\mathbf{x}|^{3}}\left(\begin{array}{ccc}
0 & -z & y \\
z & 0 & -x \\
-y & x & 0
\end{array}\right),
$$

is the Biot-Savart kernel and $\eta$ is a cutoff parameter. The velocity field at $\mathbf{x}_{i}$ due to the $j$ th sheet is then determined from

$$
\left[\int_{V_{j}} K_{\eta}\left(\mathbf{x}_{i}-\mathbf{x}^{\prime}\right) d \mathbf{x}^{\prime}\right] \boldsymbol{\Omega}_{j}\left(\mathbf{x}_{j}, t\right)
$$

since $\boldsymbol{\Omega}_{j}\left(\mathbf{x}_{j}, t\right)$ is taken to be constant over the sheets. Here, $V_{j}$ represents the volume of the $j$ th sheet. In view of the high aspect ratio of the vortex sheets, the $y$ integration in (11) can be approximated by a midpoint rule. Furthermore, the $x$ and $z$ integrals can be done analytically for all values of $\mathbf{x}_{i}$ and $\mathbf{x}^{\prime}$. In general,

$$
\int_{V_{j}} K_{\eta}\left(\mathbf{x}_{i}-\mathbf{x}^{\prime}\right) d \mathbf{x}^{\prime}=\left(\begin{array}{ccc}
0 & W_{i j} & -V_{i j} \\
-W_{i j} & 0 & U_{i j} \\
V_{i j} & -U_{i j} & 0
\end{array}\right) .
$$

When no part of the $j$ th sheet intersects a sphere of radius $\eta$ around the point $\mathbf{x}_{i}, K_{\eta}=K$ in (11) and

$$
\begin{gathered}
U_{i j}=-\frac{h_{j}}{8 \pi} \ln \left(\frac{r_{11}-Z_{1}}{r_{11}+Z_{1}} \frac{r_{21}+Z_{1}}{r_{21}-Z_{1}} \frac{r_{22}-Z_{2}}{r_{22}+Z_{2}} \frac{r_{12}+Z_{2}}{r_{12}-Z_{2}}\right) \\
V_{i j}=-\frac{h_{j}}{4 \pi}\left(\tan ^{-1} \frac{X_{2} Z_{2}}{Y r_{22}}-\tan ^{-1} \frac{X_{2} Z_{1}}{Y r_{21}}-\tan ^{-1} \frac{X_{1} Z_{2}}{Y r_{12}}+\tan ^{-1} \frac{X_{1} Z_{1}}{Y r_{11}}\right) \\
W_{i j}=-\frac{h_{j}}{8 \pi} \ln \left(\frac{r_{11}-X_{1}}{r_{11}+X_{1}} \frac{r_{21}+X_{1}}{r_{21}-X_{1}} \frac{r_{22}-X_{2}}{r_{22}+X_{2}} \frac{r_{12}+X_{2}}{r_{12}-X_{2}}\right)
\end{gathered}
$$


where $r_{m n}^{2}=X_{m}^{2}+Y^{2}+Z_{n}^{2}, m, n=1,2, X_{1}=\left(x_{i}-x_{j}-l\right) / \eta, X_{2}=\left(x_{i}-x_{j}+l\right) / \eta, Z_{1}=\left(z_{i}-z_{j}-d\right) / \eta$, $Z_{2}=\left(z_{i}-z_{j}+d\right) / \eta$ and $Y=\left(y_{i}-y_{j}\right) / \eta$. A geometric interpretation of $r_{m n}$ is given in Fig. 3 .

In view of the high aspect ratio of the sheets, the condition $\eta<l, d$ is always satisfied so that no sheet can fit entirely within a ball of radius $\eta$ around $\mathbf{x}_{i}$. Thus, the evaluation of $U_{i j}, V_{i j}$ and $W_{i j}$ in the local case involves carrying out the integration of $K_{\eta}$ over a region formed by the intersection of a sheet with a sphere of radius $\eta$ around the point $\mathbf{x}_{i}$. The resulting formulas are too lengthy to write down here, though a FORTRAN subroutine based on these results is available from the author.

Individual segments of a vortex filament, say between $\mathbf{x}_{j-1}$ and $\mathbf{x}_{j}$, contribute to the velocity field at $\mathbf{x}$ according to [5]

$$
-\frac{\Gamma}{4 \pi} \frac{\mathbf{r}_{j} \times \mathbf{s}_{j}}{\left|\mathbf{r}_{j}\right|^{3}} \phi(r / \sigma)
$$

where $\Gamma$ is the circulation of the filament, $\mathbf{r}_{j}=\mathbf{x}_{j}-\mathbf{x}, \mathbf{s}_{j}=\mathbf{x}_{j}-\mathbf{x}_{j-1}$ and $\phi(r)=\left(1-\left(1-\frac{3}{2} r^{3}\right)\right) e^{-r^{3}}$ is a higher order smoothing function. It follows that the velocity field due to a collection of sheets and filaments is given by

$$
\mathbf{u}(\mathbf{x}, t)=\sum_{i=1}^{N_{s}}\left(\begin{array}{ccc}
0 & W_{i} & -V_{i} \\
-W_{i} & 0 & U_{i} \\
V_{i} & -U_{i} & 0
\end{array}\right) \boldsymbol{\Omega}_{i}-\frac{1}{4 \pi} \sum_{i=1}^{N_{f}} \frac{\mathbf{r}_{i} \times \mathbf{s}_{i}}{\left|\mathbf{r}_{i}\right|^{3}} \Gamma_{i} \phi(r / \sigma) .
$$

For flat plate boundary layer or channel flows, the sum in (16) includes a set of image sheets and filaments enabling the non-penetration boundary condition to be satisfied identically. For more general geometries, a separate calculation of a potential flow using boundary elements [7] may be added to (16) in order to satisfy the wall normal boundary condition.

For large enough values of $\left|\mathbf{x}_{i}-\mathbf{x}_{j}\right|$, the velocity corresponding to (12)-(14) converges to the velocity field associated with a vortex segment having circulation $\Gamma$ and length $\Delta s$ such that

$$
\Omega V=\Gamma \mathbf{s}
$$

where

$$
\mathbf{s}=\frac{\mathbf{\Omega}}{|\boldsymbol{\Omega}|} \Delta s,
$$

and $V=2 l \cdot 2 h \cdot 2 d$. From (17) and (18) it follows that

$$
|\Omega| V=|\Gamma| \Delta s
$$

Since $\Gamma$ and $\Delta s$ always appear in (15) as a product $\Gamma \Delta s$, when converting a sheet to a tube, $\Delta s$ can be chosen arbitrarily - with $\Gamma$ then determined from (19). Acceptable accuracy in replacing (12)-(14) by the equivalent tube formula satisfying (17)-(19) occurs for $\left|\mathbf{x}_{i}-\mathbf{x}_{j}\right|>.5$. Since (15) is considerably cheaper to evaluate than (12)-(14), use of this equivalence is done whenever possible. This results in a substantial speed-up of the calculation.

The intersection of boundaries with the support of the smoothing function in (11) must be specifically compensated for to prevent a loss of information in the velocity field calculations. An effective technique in this regard, which is compatible with the implementation of the boundary conditions, is to force sheets within $\eta$ of the wall to contribute to the velocity at points between themselves and the wall surface according to the far field formulas (12)-(14) only. This can be done by reducing $\eta$ for these sheets. In the case of the streamwise velocity, for example, the wall sheet lying immediately over a point on the wall contributes the amount $-h\left(\Omega_{3}\right)_{i}$ to the velocity 
at this location, i.e., exactly what is expected from a physical analysis of the local vortex sheet. This result is a consequence of the difference in arctangent terms in (13) which will in this case be essentially $2 \pi$. A similar results holds for the spanwise velocity where the sheet above a point contributes $h\left(\Omega_{1}\right)_{i}$.

The boundary values of the components of vorticity tangential to the wall may be determined by setting (16) to zero at the location of each wall sheet. For planar walls containing $M$ fixed sheets on the wall, this yields two sets of $M$ coupled equations which are readily solved by iteration.

\section{Filament formation}

A critical aspect of the method is in providing for the generation of new vortex tubes. The algorithm must at the same time be both sensitive to the physical process by which new structures appear, yet not so unconstrained as to allow for the formation of impossibly large numbers of new vortices. The assumption that new filaments are created primarily during ejection events orchestrated by pre-existing parent vortices, provides a basis for satisfying these criteria. In particular, only the most intense outward movements of vorticity may be considered to be of dynamical interest, i.e. associated with the self-replication process. The remainder of the outwardly migrating vorticity may be discarded under the assumption that it contributes only to the random background vorticity and is not dynamically essential to the turbulent flow field.

Numerical implementation of these ideas can be effected through the following considerations. The outside of the sheet region is at $y^{*}$ and beyond this point the vorticity resides exclusively in vortex tubes. At each time step, after the sheets are convected according to (2), those sheets in the furthest layer above the wall, and for which $v>0$, will have moved past the plane $y=y^{*}$. The regridding step which updates the vorticity in the sheets does not affect the vorticity residing above $y=y^{*}$. This vorticity, however, is potentially the source of the vorticity composing the new vortex filaments. Its appearance above the $y=y^{*}$ plane is only in spatially intermittent regions where ejections occur. Wherever this vorticity exceeds a threshold, i.e. $|\boldsymbol{\Omega}|>|\boldsymbol{\Omega}|_{\text {crit }}$ it can be formed into new filaments using (17)-(19). This limits the new vortices to only the most energetic ejection motions associated with parent vortices.

The physical events leading to a new vortex generally occurs over a time interval larger than a single numerical time step. In other words, for a number of consecutive time steps a single parent vortex may eject a series of filaments at a particular sheet location-each exceeding the threshold and virtually identically in direction and amplitude to those preceding it. Rather than permit each of these filaments to be individually represented in the calculation, it is most efficient to collect them together into a single structure of greater circulation. This may be done by adding the amplitudes of the new filaments to the pre-existing ones and updating the location of the combined vortex through a weighted average depending on the relative strength of the two component vortices. If the developing filament convects more than the distance $l$ downstream of its original point before the creation process is complete, it is then considered in its final form and a new vortex is initiated at the source location.

\section{$5 \quad$ Numerical results}

Calculations performed to date have focused on simulating turbulent channel flow so as to be able to systematically document the capabilities of the vortex method in reproducing the principal statistical and physical attributes of turbulence. The channel flow is defined by holding fixed the average centerline streamwise velocity (scaled by the average mass flow velocity) at $U_{c l}=1.165$, 
corresponding to the value computed in a spectral DNS of channel flow at $R_{\tau}=U_{\tau} H / \nu=250$, where $U_{\tau}$ is the friction velocity and $H$ is the channel width. The computational domain is $2.5 \times$ $1 \times 2.5$ units in the streamwise, wall-normal and spanwise directions, respectively, where $y=.5$ is the centerline of the channel. In wall units it is $625 \times 250 \times 625$. The initial grid of sheets on each wall is $16 \times 10 \times 16$ for a total of 5120 . For these, $l=d=.135$ while $h=.016$, so that the sheets cover only the regions $0 \leq y \leq .144$ and $.856 \leq y \leq 1$. In terms of wall variables, the sheets have dimension $39.1 \times 4.03 \times 39.1$. The initial vorticity distribution in the sheets is taken to be that corresponding to the mean vorticity profile in the channel flow simulation. To perturb this one-dimensional field, either a single tilted vortex is used or else a collection of 18 vortices found from a spectral calculation. In either case the subsequent evolution of the flow has similar properties.

Calculations have been performed for approximately 2000 time steps covering an elapsed time of $t \approx 8$. Over this time interval, the original structures convect through the periodic flow domain almost 4 times. An anti-symmetry condition between the vortices in the top and lower halves of the channel is used in these computations in order to enhance efficiency. Current studies, which seek to investigate the long time behavior of the algorithm, have dropped this assumption.

Since the initial spanwise vorticity is confined to just the sheet region, the flow is lacking the considerable amount of spanwise vorticity which would be present in the central region of the channel in the fully developed turbulent state. The computed solution reacts to this condition over several hundred time steps through a prodigious infusion of new vortex filaments into the flow. After several hundred new filaments appear, the conditions causing the creation of large numbers of vortices changes, and the rate of introduction of new filaments slows dramatically. These points are illustrated in Fig. 4 showing the history of the number of vortex filaments and segments during the calculation. After an incubation period dominated by significant viscous diffusion of vorticity into the sheets and its outward convection, a steep rise in the number of new filaments appears. Later, the growth in the number of filaments settles down to a gentle slope. Due to vortex stretching, which increases the length of filaments, the number of segments in the calculation rises at a somewhat faster rate than the number of new filaments. Fig. 5 shows how the number of sites producing new vortices changes with time. A rapid rise is followed by an equally rapid decline to a small persistent number of isolated locations.

The evolution of the vortical structure of the channel flow from its initial state is illustrated in Figs. $6 \mathrm{a}$ and $6 \mathrm{~b}$ giving a plan view of the vortex system at two different times. Flow is from bottom to top. The new vortices are seen to form in sequence as a parent vortex convects along the wall. Due to periodicity, the regions of new vortices eventually form columns in the planar view. Three distinct arrays of new filaments are shown in Fig. 6a. While the computation displayed here started with a wide distribution of parent vortices, a very similar picture also emerges if just a single tilted vortex is used to perturb the flow. In this case new filaments also form into three columns. This suggests the presence of a significant spanwise instability in the initial conditions.

As time progresses (Fig. 6b) the regions of new filaments become wider and an interesting streamwise instability appears as the solution evolves toward a more chaotic state. The new filaments consist of hairpins together with spanwise vortices taking on an increasing quasi-streamwise orientation as time progresses. Seen from the side in Fig. 7, the quasi-streamwise structure of the vortex array is evident. This figure also reveals the presence of a significant ejection of vorticity towards the centerline. The difference between the initial mean velocity field and that corresponding to the state achieved in Figs. $6 \mathrm{~b}$ and 7 is given in Fig. 8. This shows just how much the flow has evolved toward a turbulent-like state with a more physical distribution of vorticity.

It is informative to examine the relationship between the numerical and physical vortex creation process in some detail. In particular, it is of interest to see how the former accommodates the latter 
through the generation of collections of individual filaments. The process as it is manifested in the calculations performed thus far appears to be consistent with many previous observations $[2,4,10]$. A particular scenario is illustrated in Figs. 9-11 showing the interaction of a single tilted quasistreamwise vortex with a mean turbulent field. The vortex is initially of length .5 and is tilted at $16^{\circ}$ inclination. Figures $9 \mathrm{a}-\mathrm{c}$ show a plan view of the vortex together with the $\Omega_{x}, \Omega_{y}$ and $\Omega_{z}$ vorticity contours, respectively, at $y^{+}=39$. The scene is shortly after new vortices have begun to appear. Five new filaments are discernible in the figures, indicated by the line segments joined by circles. The pre-existing vortex is indicated by plus signs. Figure $9 \mathrm{c}$ shows that there is a region of high and low $\Omega_{z}$ on either side of the parent vortex. Enhanced, i.e. more negative vorticity is on the left side of the structure coming from the upwelling of high $\Omega_{z}$ fluid from below. To the right of the vortex, $\Omega_{z}$ is depressed in magnitude by motion bringing irrotational fluid toward the wall.

The patterns of $\Omega_{x}$ and $\Omega_{y}$ contours in Figs. 9a and b correlate with the conceptualization shown in Fig. 10. In particular, ejection of fluid from near the wall is accompanied by a bending of vortex lines in the $y-z$ plane. A notable feature of this is a pattern of + and $-\Omega_{y}$ contours as may be observed in Fig. 9b. By forward shearing of the ejecting vorticity, a + and - pattern of $\Omega_{x}$ contours also occurs as is visible in Fig. 9a.

New filaments appear at locations where the 'ejected' vorticity (i.e. vorticity which is convected out beyond the level $y=y^{*}$ ) exceeds a threshold. The five new vortex filaments in Fig. 9 occupy positions equivalent to the large arrows in Fig. 10. In the subsequent time interval such filaments get sheared, creating counterrotating vortices to the parent. Hairpin-like vortices are also produced if the new filament straddles the maximum ejection point. The vortex filament creation process some time later is shown in Figs. 11a (planar view) and 11b (side view). The field of new vortices spawned by the parent are grouped together forming a structure which is very reminiscent of those observed by Miyake and Tsujimoto [10]. From the side it is seen that the new vortex is above the parent and is composed of transverse, quasi-streamwise and hairpin filaments. The newest filaments are being added on the downstream end as the parent vortex convects through the flow. Finally, it should be remarked that other scenarios of vortex creation may also be possible depending on the initial vortex configuration.

\section{Conclusions}

A three-dimensional vortex sheet and filament method has been constructed for simulation of turbulent flow. New filaments are created during significant ejection events initiated by parent vortices. Chorin's hairpin removal algorithm is used as a physically based subgrid model to limit the range of resolved scales. Numerical studies of the development of a channel flow with particular emphasis on the vortex self-replication process have been made.

From a perturbed state, the numerical solution is observed to develop toward a self-sustained turbulent flow. An initial explosive growth in the number of new vortex structures occurs as the flow compensates for the absence of sufficient spanwise vorticity in the initial conditions. Vortex production is self-limiting, however, and the flow subsequently evolves toward a quasi-equilibrium state where new vortex production occurs at only a small number of spatially intermittent locations. Preliminary statistics taken from ongoing simulations suggest that the computational field is approaching the correct physical state.

Long time calculations of channel flow must be completed to verify that the method is able to accurately model real turbulent flows. In view of the ease with which the code can be modified to accommodate complex geometries, preparations are also being made for simulating an external flow. In particular, implementation of the scheme for the case of flow past a $6: 1$ prolate spheroid 
is planned for the near future. The intent is to get an early indication of the effectiveness of the methodology in design work.

\section{Acknowledgement}

This research was supported by ONR Grant N00014-93-10184. Computations were performed on the Maui HPCC IBM-SP computer.

\section{References}

[1] Bernard, P.S., "A Deterministic Vortex Sheet Method for Boundary Layer Flow," J. Comp. Phys., 117, pp. 132, 1995.

[2] Bernard, P.S., Thomas, J.M. and Handler, R.A., "Vortex Dynamics and the Production of Reynolds Stress," J. Fluid Mech., 253, pp. 385, 1993.

[3] Bernard, P.S. and Thomas, J.M., "A Deterministic Vortex Sheet Method for Three-Dimensional Boundary Layers," Sandia Labs Forum on Vortex Methods for Engineering Applications, February, 1995.

[4] Brooke, J.W. and Hanratty, T.J., "Origin of Turbulence-Producing Eddies in a Channel Flow," Phys. Fluids A, 5, pp. 1011, 1993.

[5] Chorin, A.J., "Hairpin Removal in Vortex Interactions II," J. Comp. Phys., 107, pp. 1, 1993.

[6] Greengard, L., "The Core Spreading Vortex Method Approximates the Wrong Equation," $J$. Comp. Phys., 61, pp. 345, 1985.

[7] Hess, J.L. and Smith, A.M.O., "Calculation of Potential Flow about Arbitrary Bodies," Prog. Aero. Sci., 8, pp. 1, 1967.

[8] Leonard, A., "Numerical Simulation of Interacting, Three-Dimensional Vortex Filaments," Lec. Notes in Phys., 35, pp. 245, 1975.

[9] Leonard, A., "Vortex Methods for Flow Simulation," J. Comp. Phys., 37, pp. 289, 1980.

[10] Miyake, Y. and Tsujimoto, K., "Behavior of Quasi-Streamwise Vortices in Near-Wall Turbulence," Proc. ASME Fluids Engineering Division Summer Meeting, FED - Vol. 238, Part 3, pp. 41-48, 1995.

[11] Puckett, E.G., "Vortex Methods: An Introduction and Survey of Selected Research Topics," in Incompressible computational fluid dynamics: trends and advances, edited by M.D. Gunzburger and R.A. Nicolaides, Cambridge University Press, pp. 335-408, 1993.

[12] Rossi, L.F., "Resurrecting Core Spreading Vortex Methods: A new Scheme that is Both Deterministic and Convergent," SIAM J. Sci. Comp., 17, pp. 370, 1995. 

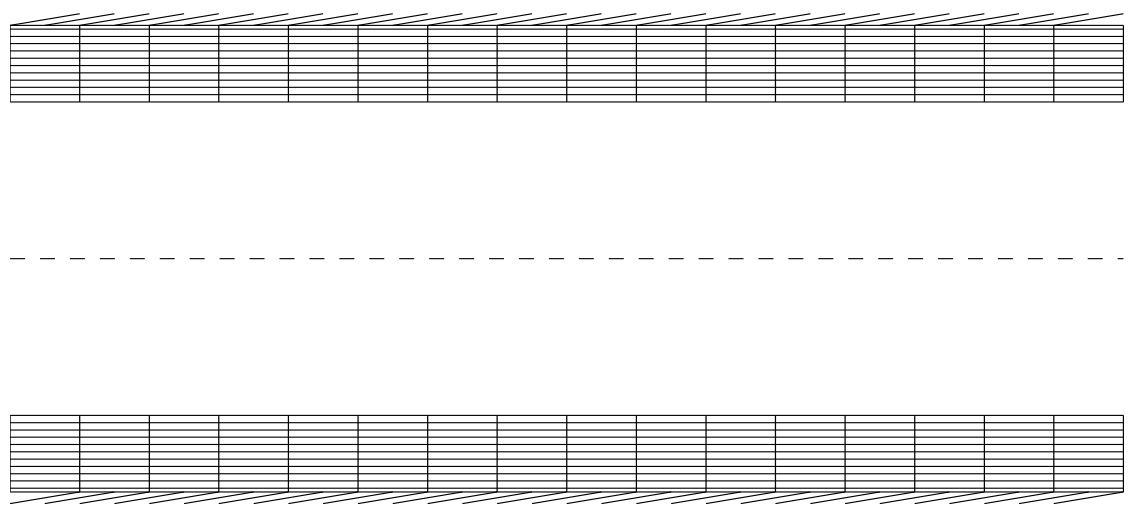

Figure 1: Sheet arrangement in channel flow.

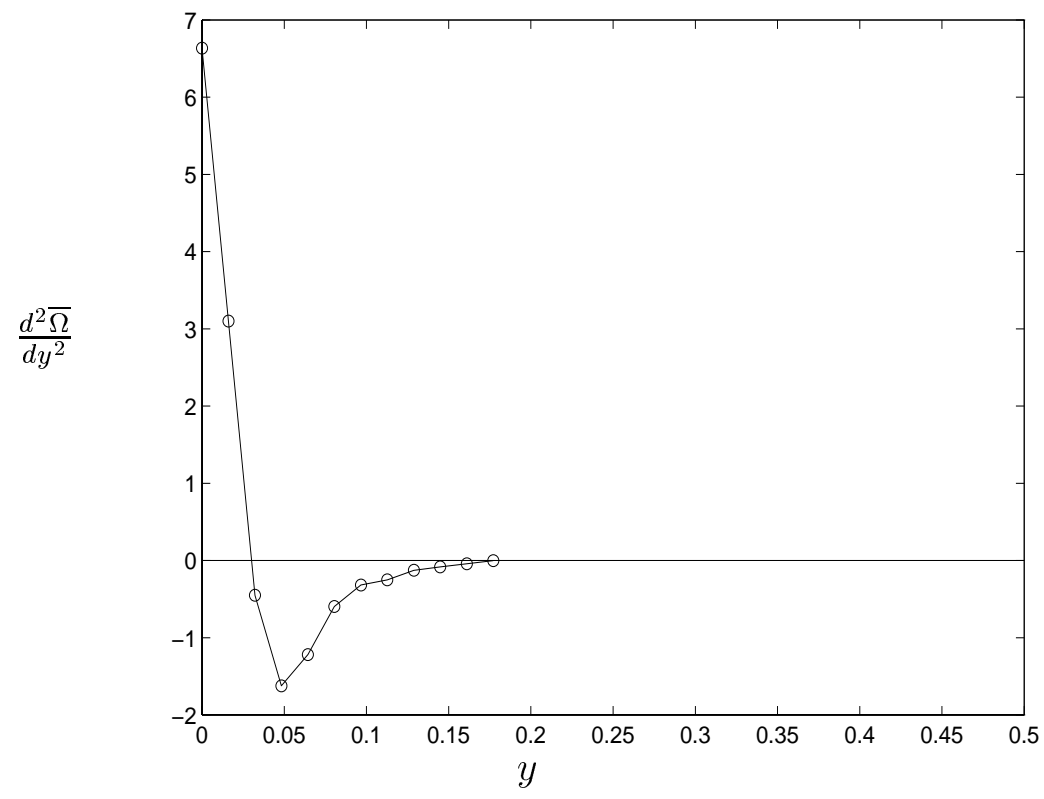

Figure 2: The extent of mean viscous diffusion. 


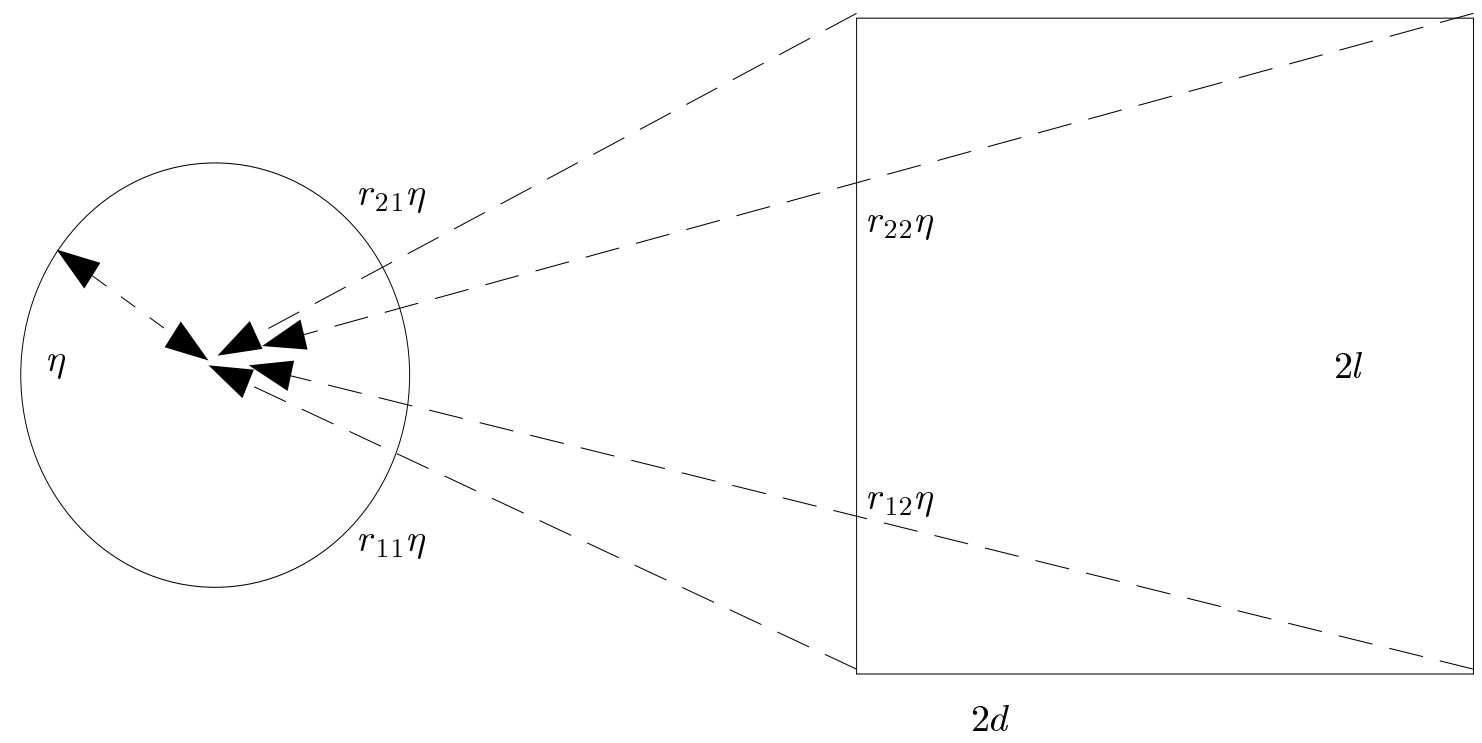

Figure 3: Geometry of sheet interactions.

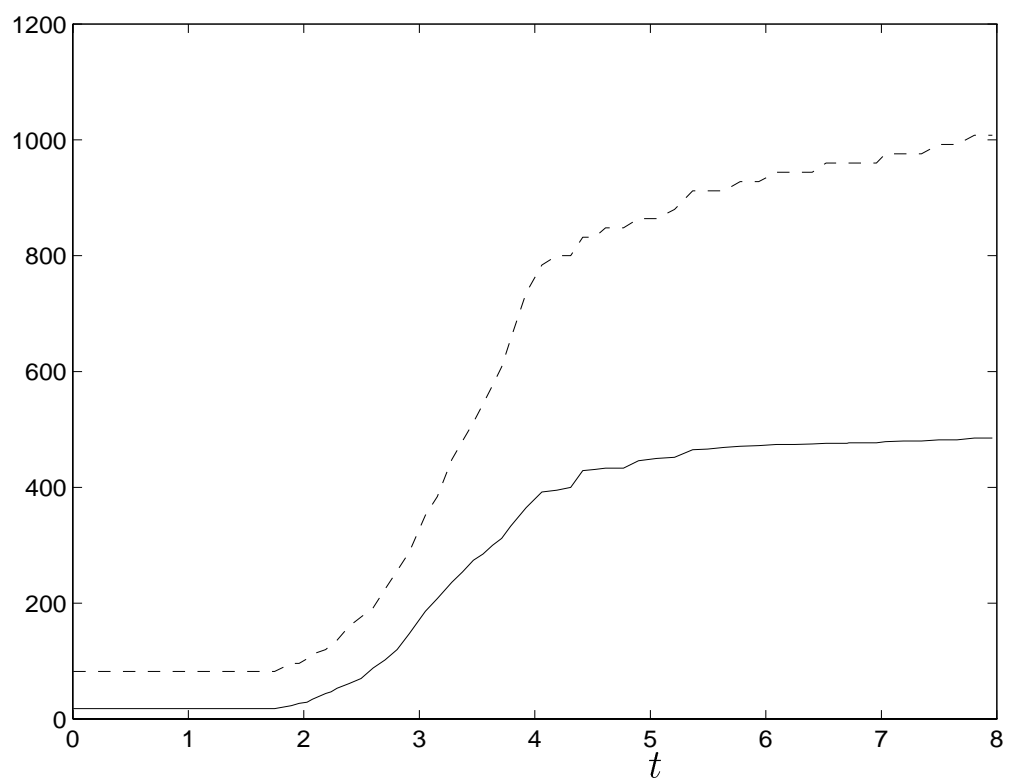

Figure 4: Number of vortex filaments (solid line) and segments (dashed line) during the calculation. 


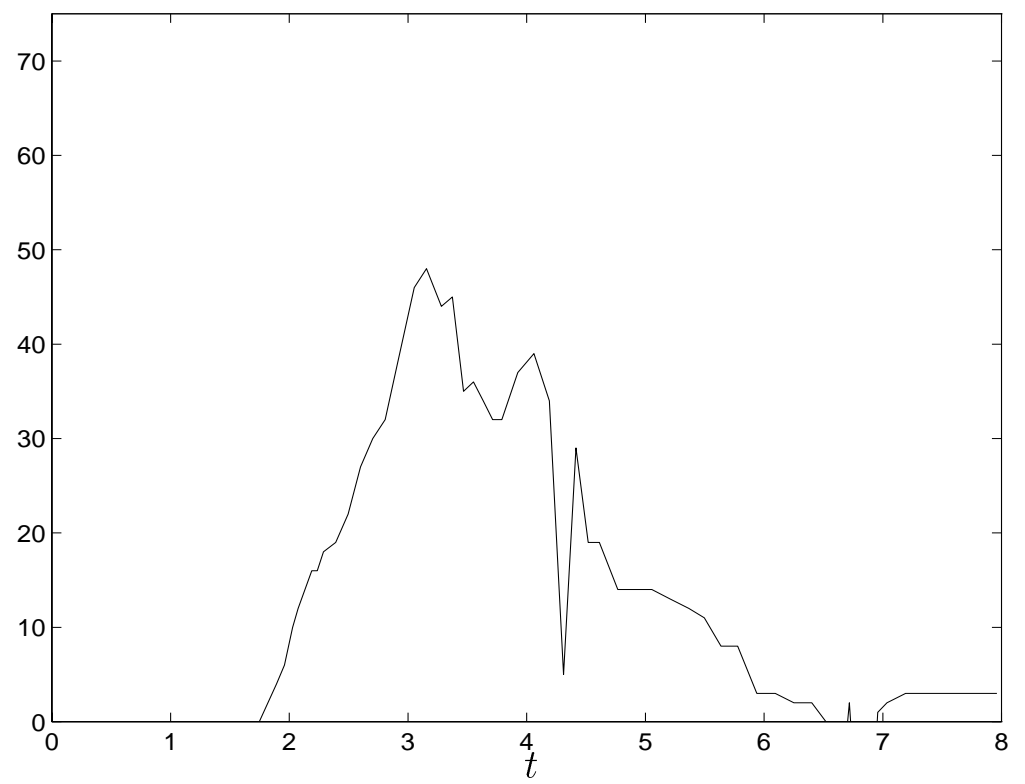

Figure 5: Number of sites producing vortices. 
(a)

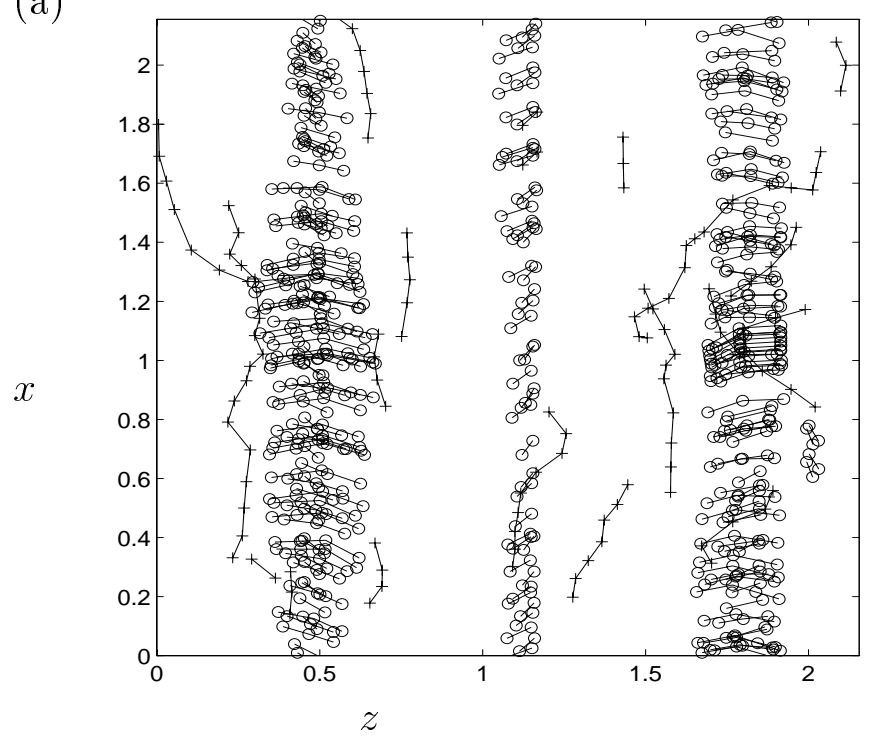

(b)

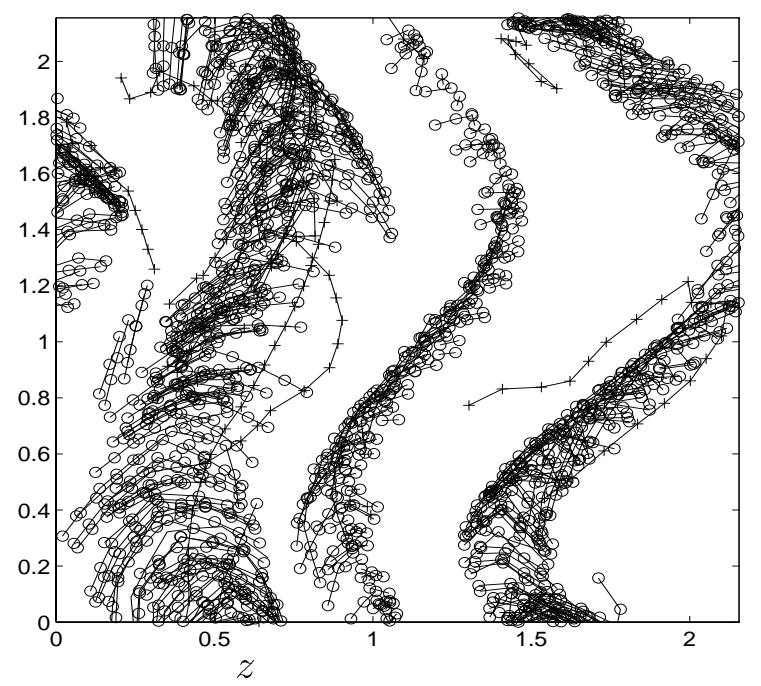

Figure 6: Early (a) and later (b) stages in the developing vortex filament field. 


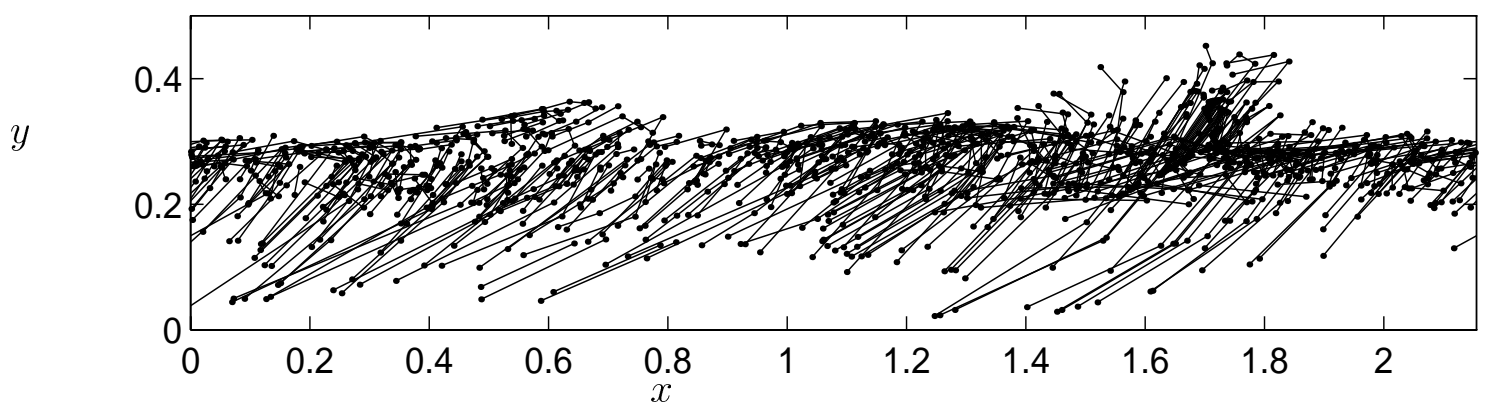

Figure 7: Side view of the developing vortex filament field.

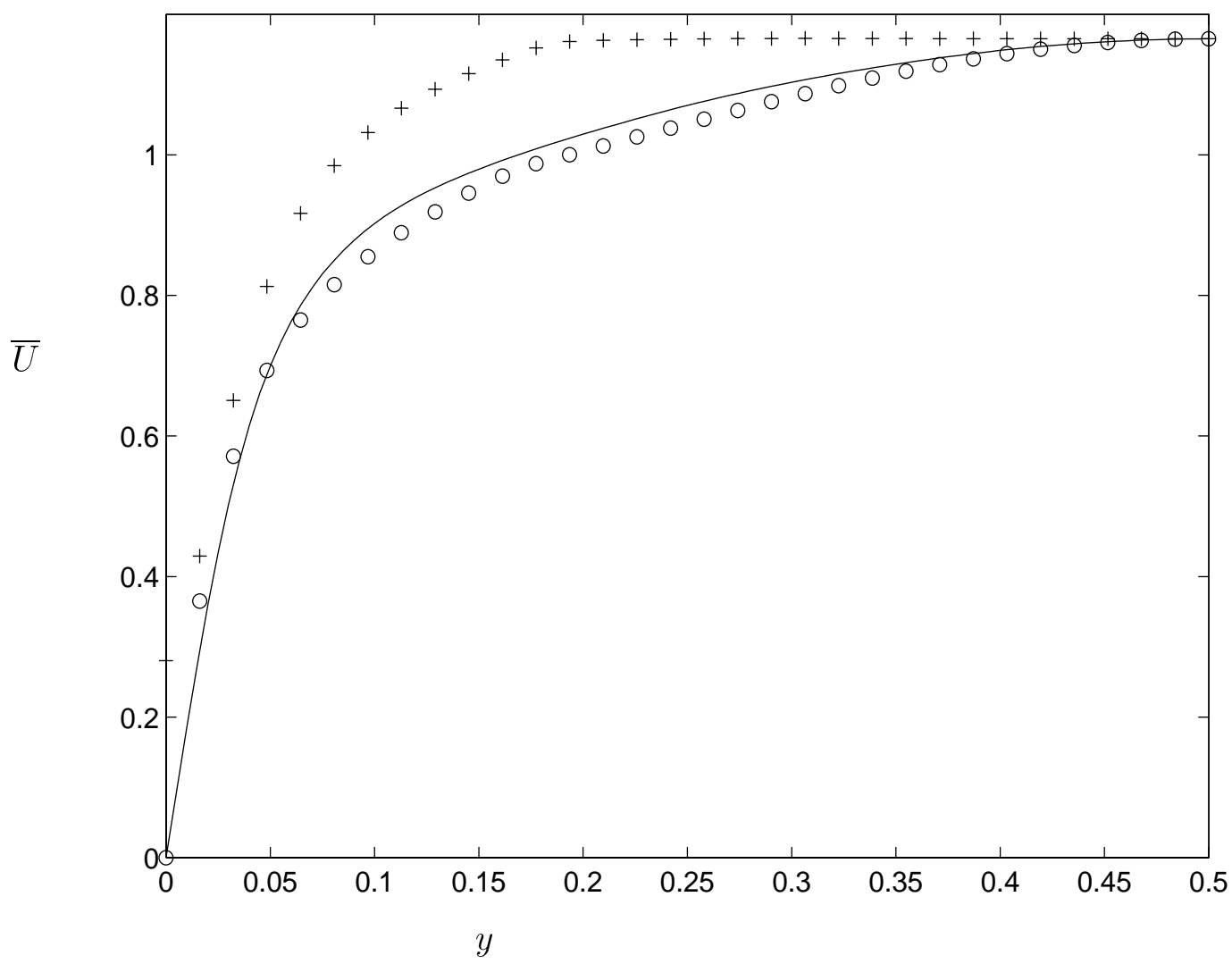

Figure 8: Mean velocity distribution. +, initial condition; - DNS; and o, mean velocity field corresponding to Figs. 6b and 7. 
(a)

$x$

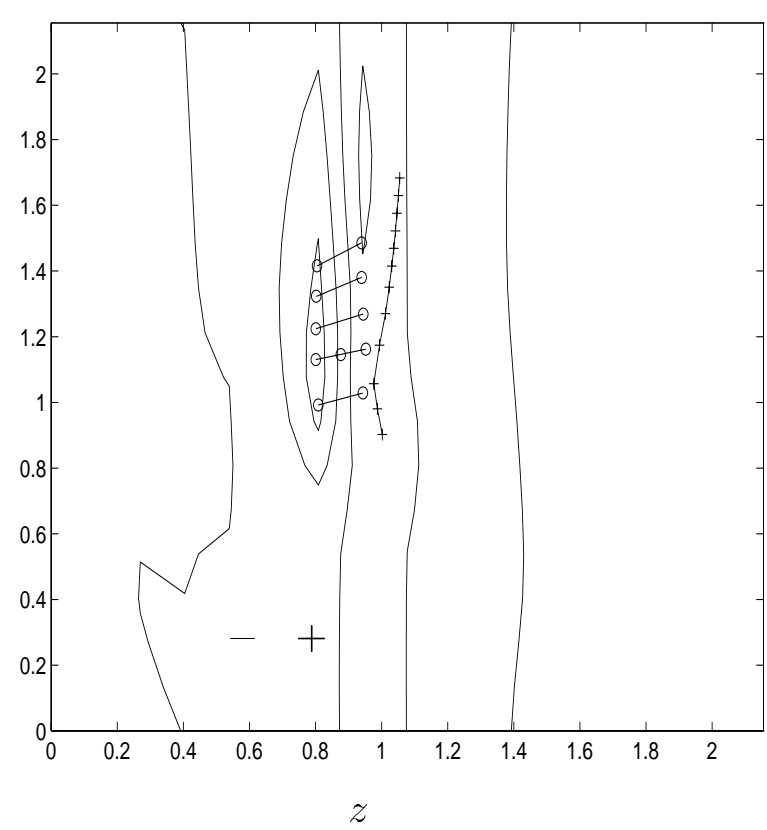

(b)

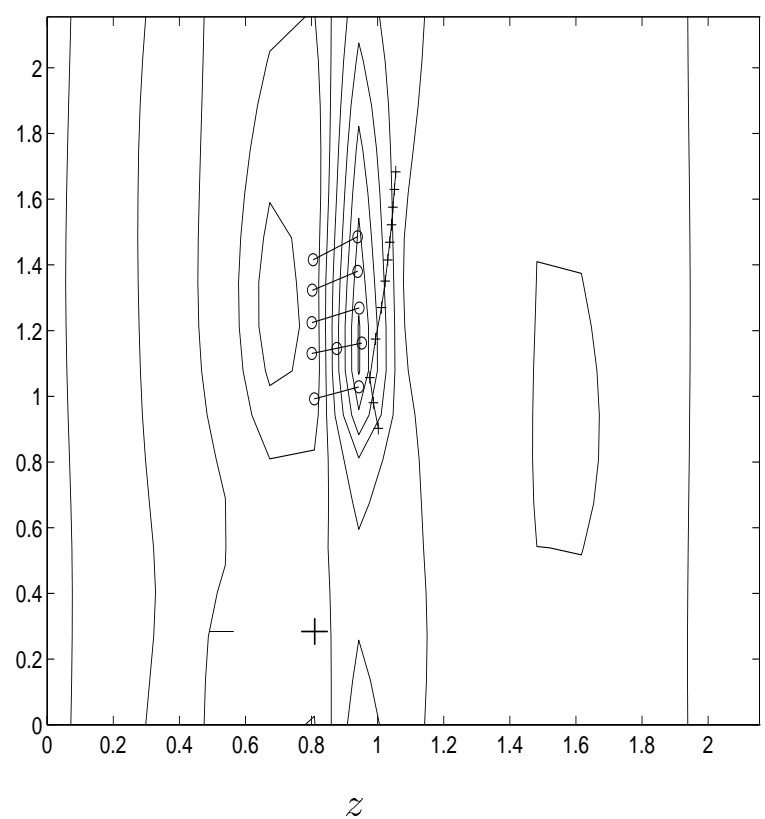

Figure 9: Vorticity contours at $y^{+}=30,+$, initial vortex; o, new filaments. (a) $\Omega_{x}$, (b) $\Omega_{y}$, (c) $\Omega_{z}$. 


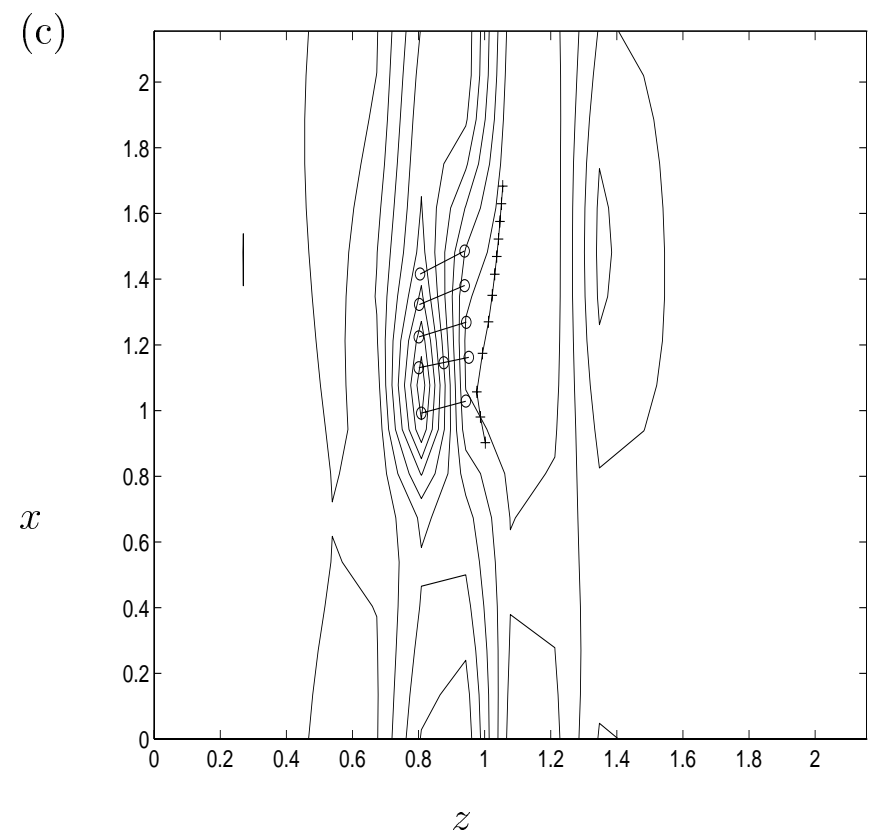

Figure 9: (Continued) Vorticity contours at $y^{+}=30,+$ initial vortex; o new filaments. (a) $\Omega_{x}$, (b) $\Omega_{y}$, (c) $\Omega_{z}$.

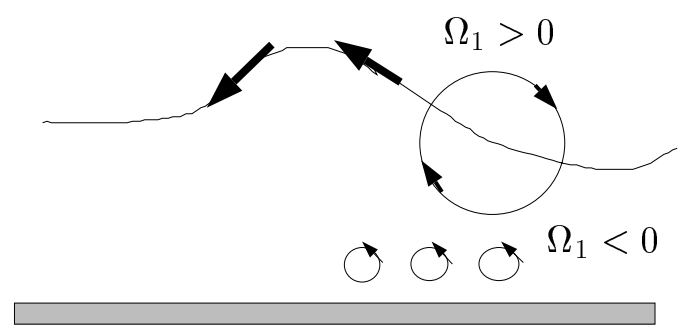

Figure 10: Conceptualization of self-replication process. Flow is into the page. 
(a)

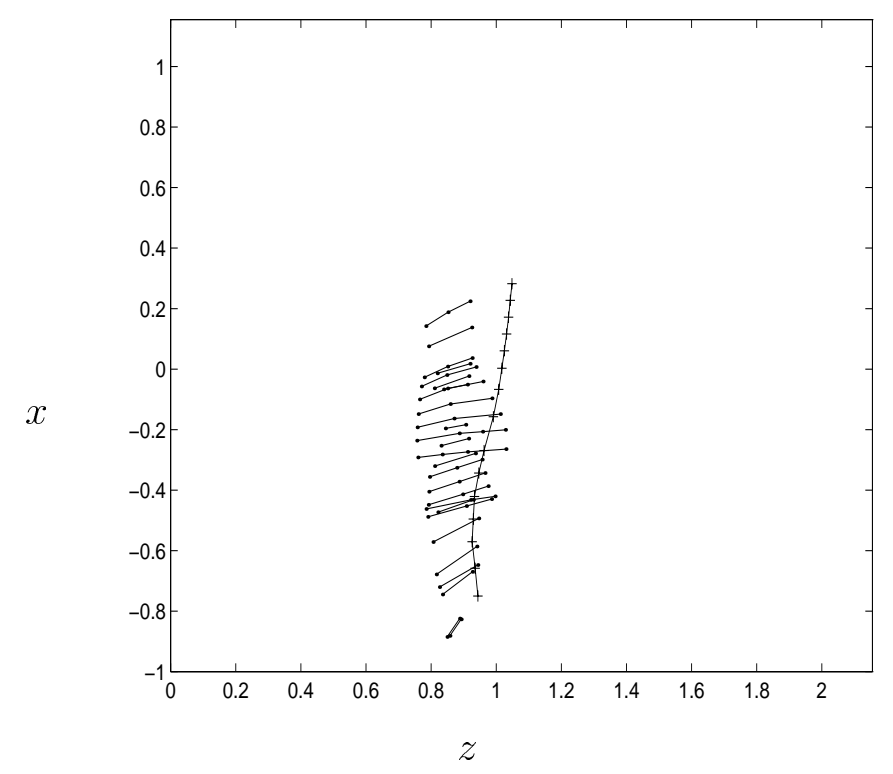

(b)

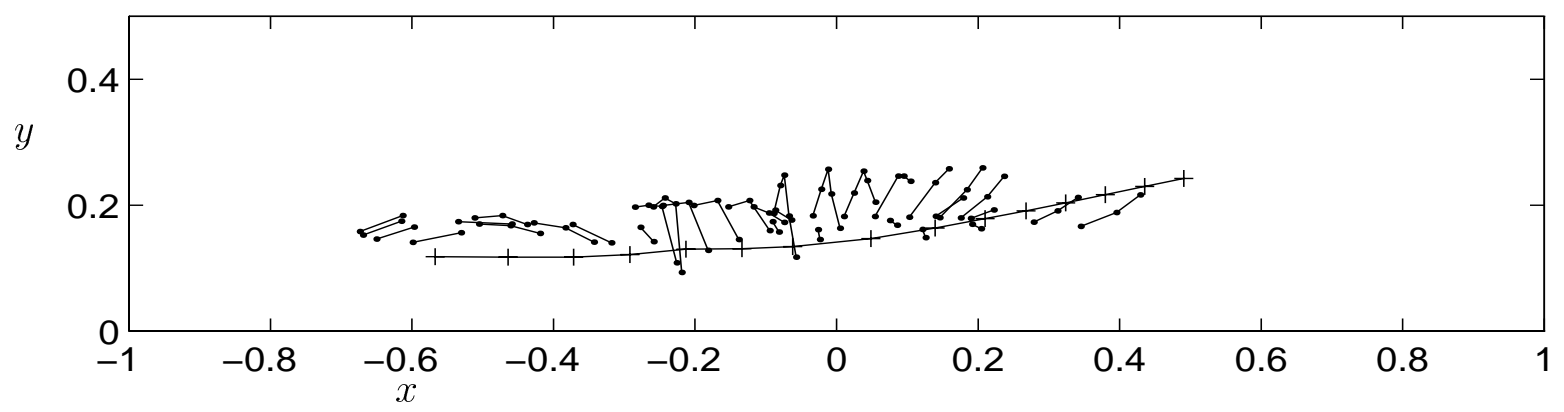

Figure 11: Vortex self-replication: +, parent vortex and new filaments. (a) plan view, (b) side view. 